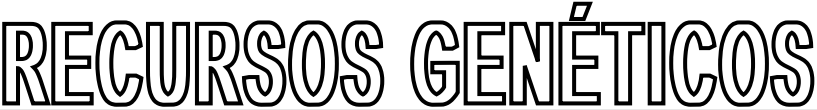

\section{GERMOPLASMA DE TOMATE SILVESTRE (Lycopersicum spp.) EN LA RESERVA DE RECURSO GENÉTICOS DE APACUNCA (RRGA), CHINANDEGA}

\section{WILD TOMATO GERMPLASM (Lycopersicum spp.) IN THE RESERVE OF GENETIC RESOURCES OF APACUNCA (RRGA) CHINANDEGA}

\section{Benavides González Alvaro ${ }^{1}$, Cisne Contreras José ${ }^{1}$, Querol Lipcovich Daniel ${ }^{2}$, Morán Centeno Juan Carlos ${ }^{1}$} ${ }^{1}$ Grupo DEPARTIR, FAGRO. Álvaro.Benavides@una.edu.ni

${ }^{2}$ Asesor y consultor del grupo DEPARTIR.

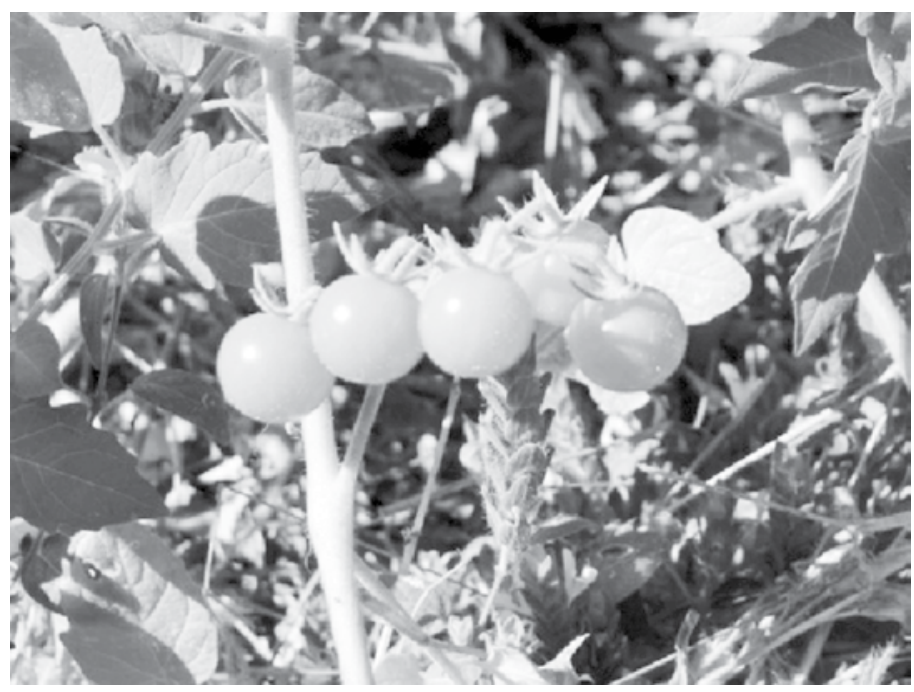

\section{RESUMEN}

Considerando la importancia del tomate como cultivo hortícola, el presente estudio se realizó con el fin de aportar información in situ y ex situ usando seis muestras de poblaciones d tomate silvestre (Lycopersicum spp.), endémicas de la Reserva de Recursos Genéticos de Apacunca (RRGA). Se implementaron normas de riego $(0.4,0.7 \mathrm{y}$ 1.1 litros/planta/día) y niveles de biofertilizante $(0,100$, 200 y $300 \mathrm{cc}$ por bombada de 20 litros). Los experimentos establecidos ex situ fueron arreglados en diseños en franjas con tres réplicas y se utilizaron análisis univariados y multivariados en datos morfológicos. Los estudios in situ y ex situ indican que las poblaciones difieren en algunas variables, en donde variables de frutos y de planta fueron afectadas de manera significativa por las normas de riego y los niveles de biofertilizante, respectivamente. La mayor cantidad de frutos se obtuvieron con la dosis intermedia de riego, y los mayores niveles de biofertilizante. Los primeros tres componentes principales aislaron el $73.2 \%$ de la variación total, y las dimensiones del fruto, longitud de tallo y entrenudos en la planta fueron las variables discriminantes entre los

\begin{abstract}
Considering the importance of tomato as a vegetable crop, the present study was carried out to provide in situ and ex situ information using six samples from populations of wild tomato (Lycopersicum spp.), those samples are endemic in Reserve of Genetic Resources of Apacunca (RRGA). Irrigation indices were implemented $(0.4,0.7$ and 1.1 liters / plant / day) and biofertilizer levels $(0,100,200$ and 300 cc per pump of 20 liters of content). Ex situ experiments were arranged in split-block design with three replicates using univariate and multivariate analysis on morphological data's. In situ and ex situ studies revealed differences among populations in some variables, fruit and plant variables were significantly affected by the irrigation indices and biofertilizer levels, respectively. The largest amount of fruits was obtained with the intermediate irrigation index and high levels of biofertilizer. The first three principal components isolated the $73.2 \%$ of the total variation, and the discriminate variables were established by the fruit dimensions, stem and internode length. The populations evaluated in situ, with irrigation indices and biofertilizer levels formed three distinct cores.
\end{abstract}


tratamiento conformados. Las poblaciones evaluadas in situ, con normas de riego y niveles de biofertilizante conformaron tres núcleos bien diferenciados. Las mediciones realizadas en las poblaciones en su hábitat natural (in situ) fueron menos variantes, en comparación con las poblaciones ex situ y expuestas a tratamientos. Las características encontradas en el tomate silvestre de la Reserva, sugieren que puede ser utilizado en la alimentación de las familias rurales, por lo tanto, se debe asegurar su conservación in situ y ex situ. Palabra clave: Lycopersicum spp, diseños en franjas, normas de riego y biofertilizantes.
Measurements performed on the populations in their natural habitat (in situ) showed less variation in comparison to ex situ population and exposed to the treatments. The characteristics found in the wild tomato from Reserve, suggest than this wild crop can be used as feed in the rural households, therefore, is necessary their in situ and ex situ preservation. Keywords: Lycopersicum spp., split-block designs, irrigation, biofertilizers, wild germplasm.
$\mathrm{E}$ 1 conocimiento de la biodiversidad en un área determinada es de suma importancia para su conservación dentro de las áreas protegidas o dentro de las biosferas; sin embargo, no se tiene dicho listado y cuando existe al menos una parte, éste no presta la menor atención a los parientes silvestres de las plantas cultivadas. Ante esta situación es recomendable la elaboración de un inventario detallado de las especies silvestres emparentadas con las cultivadas que están bajo conservación en las áreas protegidas (Azurdia, 1996b). Todo esto permitiría que en el futuro se realice el monitoreo frecuente de dichas especies para asegurarse de que están siendo conservadas eficientemente y disponibles para los fitomejoradores (Meyrat, 2001).

El tomate (Lycopersicum esculentum) es una de las hortalizas de mayor importancia, por lo que es necesario el estudio de sus parientes silvestres, dichas especies silvestres son diploide con 24 cromosomas en sus células somáticas.

Carrillos y Chávez (2010), mencionan que los actuales programas de mejoramiento genético buscan además de mejorar la productividad y adaptación, incorporar alta calidad nutricional, resistencia a patógenos, para obtener materiales apropiados mediante la introgresión, o retrocruzamiento. Por otro lado, el IPGRI (2000), indica que antes de promover la utilización de especies, es necesario conocer su variabilidad genética conservada en su hábitat natural, por lo que se debe prestar atención a las especies silvestres, realizar inventario y su descripción.

Considerando que el tomate tiene su origen en Latinoamérica, es muy importante el estudio de esta especie en su estado silvestre, así como expuesta a tratamientos.

\section{MATERIALES Y MÉTODOS}

Lugar de estudio. La Reserva de Recursos Genéticos de Apacunca (RRGA) forma parte del Corredor Biológico Mesoaméricano (CBM), se encuentra ubicada en el departamento de Chinandega en el extremo nor-occidental de la macro región del Pacífico. Limita al norte con Honduras y el departamento de Madriz, al sur con el
Océano Pacífico, al este con el departamento de León y al oeste con el Golfo de Fonseca (MAG-FOR, 1997).

En las planicies de Somotillo se encuentran las depresiones de Villanueva y Somotillo. Comprende las zonas más altas de la depresión nicaragüense, y conforman un relieve plano suavemente ondulado con pendientes menores del $15 \%$, con una asociación de suelos de texturas pesadas (vertisoles) en las áreas planas y depresionales, y suelos poco profundos de textura medias a finas en las partes más altas del relieve con drenaje imperfecto; y por su cercanía al Estero Real está sujeta a frecuentes inundaciones por el desborde de los ríos Villanueva y Negro (Levard et al., 2000).

El clima según Holdridge corresponde a zonas de vida de bosque seco tropical (bs-T) con inclusiones de bosque seco subtropical, cálido monzónico (bs-S) y $\mathrm{su}$ precipitación es de aproximadamente $1600 \mathrm{~mm}^{\text {-año. }}$ Igualmente presenta un período canicular definido, en donde la época seca ocurren el $8 \%$ de las precipitaciones $\mathrm{y}$ el $92 \%$ en época de lluvia y su temperatura media anual oscila entre los $28{ }^{\circ} \mathrm{C}$ y $29{ }^{\circ} \mathrm{C}$ (MARENA, 1999).

La localidad de Managua se ubica geográficamente a $12^{\circ} 8^{\prime}$ latitud Norte y $86^{\circ} 10^{\prime}$ longitud Oeste a una altura de $56 \mathrm{msnm}$. Los suelos pertenecen a la serie La Calera; presentan una textura franco a franco - arenosa con $\mathrm{pH}$ de 7.8 a 8.5 y una pendiente de $0-2 \%$. La zona se caracteriza por su clima tropical de sabana, caracterizado por una prolongada estación seca y por temperaturas altas todo el año, que van desde $27{ }^{\circ} \mathrm{C}$ hasta $34^{\circ} \mathrm{C}$. La precipitación anual promedio para Managua es de 1100-1600 mm, humedad Relativa de $75 \%$ y vientos de $12 \mathrm{~km} / \mathrm{h}$.

La Figura 1 muestra los promedios de temperaturas $\mathrm{y}$ totales mensuales de precipitaciones, en Chinandega y Managua. 


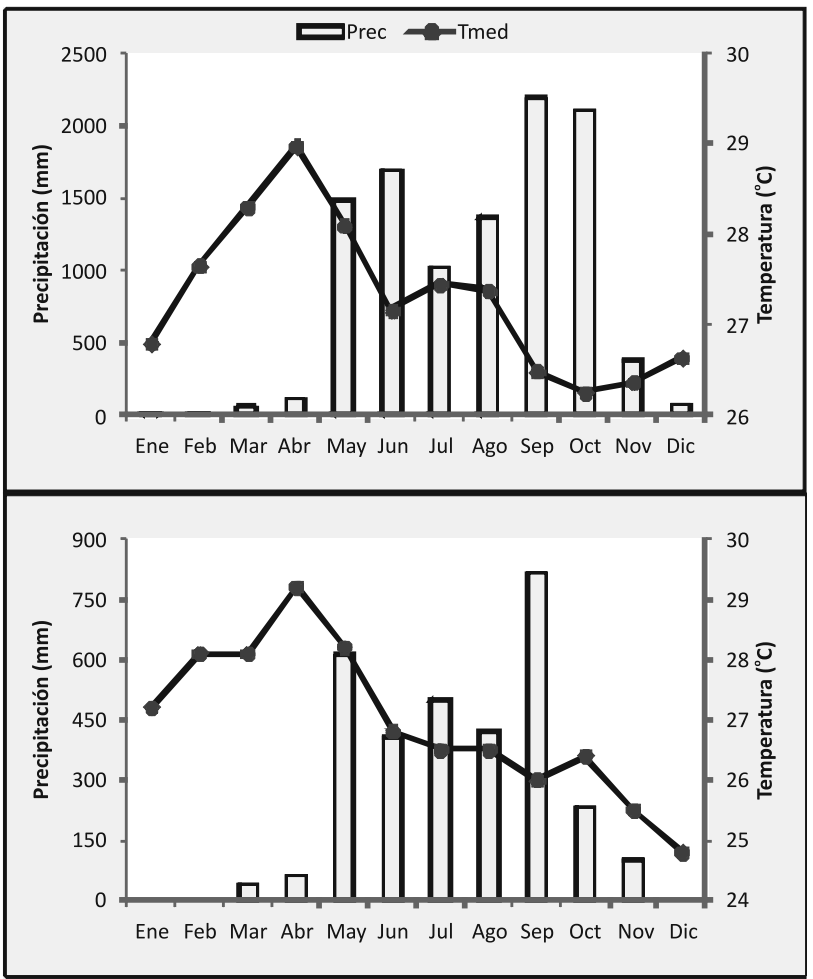

Figura 1. Condiciones climáticas de Chinandega y Managua. INETER, 2010.

El grupo de investigación Desarrollo Participativo Integral Rural (DEPARTIR) en el marco del proyecto Rescate, conservación y manejo sostenible del teocintle de Nicaragua (Zea nicaraguensis Iltis \& Benz) en la Reserva de Recursos Genéticos de Apacunca (RRGA), financiado por FAO (20102011), muestreó tomate silvestre en la zona de RRGA. Dichas poblaciones fueron estudiadas in situ, y sometidas a tratamientos. El biofertilizante a base de estiércol de ganado, suero y melaza se diluyó en agua en bomba de 20 litros, y el riego fue aplicado en litros por planta (Tabla 1).

Tratamientos y diseño experimental. Un estudio basado en submuestreos se midieron valores en variables de la especie en su hábitat natural (in situ) en marzo 2010, y en los estudios posteriores (2011) se evaluaron cuatro niveles de biofertilizantes, tres normas de riego $(0.4,0.7$ y 1.1 litros/ planta/día) y dosis de biofertilizante $(0,100,200$ y $300 \mathrm{cc}$ por bombada de 20 litros) aplicadas cada semana a las seis poblaciones (Tabla 1). En el caso del riego, se diseñó un equipo artesanal con tuberías y baldes plástico de 20 litros. Las normas de $0.4,0.7$ y 1.1 litros/planta/día fueron provistas con uno, dos y tres baldes de 20 litros, respectivamente.
Tabla 1. Ubicación geográfica de poblaciones de tomate silvestre en Apacaunca, tratamientos ex situ

\begin{tabular}{llll}
\hline Muestra & Coordenadas & Biofertilizante & Riego \\
\hline P1 & 1251588,8656116 & b0. 00 & b1. 0.4 \\
P2 & 1251903,8656342 & b1. 100 & b2. 0.7 \\
P3 & 1286448,8694936 & b2. 200 & b3. 1.1 \\
P4 & 1287461,8696199 & b3. 300 & \\
P5 & 1286456,8614929 & & \\
& 1286454,8614923 & & \\
& 1286451,8695924 & & \\
P6 & 1285811 & & \\
& 8694891 & & \\
\hline
\end{tabular}

Manejo agronómico. Los tratamientos ex situ (biofertilizante y riego) se establecieron en el área experimental de la Facultad de Agronomía, Universidad Nacional Agraria. Las semillas fueron establecidas en bandejas de polietileno de 108 orificios. Las bandejas con sustrato fue humus de lombriz a razón de $200 \mathrm{~g}$, se protegieron durante los primeros 25 días en invernadero, hubo selección de plántulas para su trasplante, una vez que alcanzaron los $20 \mathrm{~cm}$ de altura. Las distancias fueron de $1 \mathrm{~m}$ y $0.8 \mathrm{~m}$, entre surcos y planta, respectivamente. El tutoreo con material de bambú se efectuó la primera semana de marzo, en ambos ensayos; poniendo una lienza a una altura de $10 \mathrm{~cm}$ del suelo.

Análisis estadístico. En la parcela útil, fueron medidas 10 plantas de tomate por parcela. Los caracteres continuos (tallo, hoja, frutos y semillas) recopilados de los tratamientos fueron sujetos a un ANDEVA y LSD $(\infty=0.05)$ en un Modelo Aditivo Lineal propio de un diseño en franjas con tres réplicas, y analizados con SAS e InfoStat. Se determinó intervalos de confianza $(I C=\bar{y} \pm s / \sqrt{n})$. Asimismo, se estandarizaron las variables para un análisis de componentes principales a partir del método de correlaciones, y análisis de agrupamientos jerárquicos a través de método Ward y la distancia de Gower.

Análisis físico-químico de tomate silvestre. Una muestra de tomate (Maduro Firme, Estado 6 de Color USDA) silvestre de la RRGA, fue remitida al Laboratorio de Tecnología de Alimentos (LABAL), adscrito al Ministerio de Fomento, Industria y Comercio (MIFIC) del Gobierno de Nicaragua para determinar su análisis físico químico. Fue utilizado la metodología oficial propuesta por la AOAC para análisis.

\section{RESULTADOS Y DISCUSIÓN}

Tomate silvestre en Apacunca. La Reserva de Recursos Genéticos de Apacunca (RRGA) presenta diversidad genética de interés, y especies especies de alto potencial, entre las cuales se destacan el teocintle (Zea nicaraguensis Iltis \& Benz), tomate (Lycopersicum spp.), papayas (Carica spp.), cucúrbitas silvestres, entre otras especies (Benavides, 2004).

El tomate silvestre de la RRGA, también conocido como tomatillo, tomatito, tomate de monte, tomate de cerco, tomate de gallina, crece de manera silvestre en los primeros 
meses del año; pero también se cultiva en huertos familiares y los frutos se consumen frescos o son utilizados en la preparación de alimentos. En la zona, no se ha documentado ningún manejo agronómico, y la planta en ocasiones presenta síntomas de virosis y daño causados por el minador de la hoja (Lyriomiza spp.); sin embargo, la producción no cesa. Alvarez-Hernández et al., (2009), identificaron diversas especies de plagas asociadas al tomate silvestre, las cuales no obstruyeron la fructificación; asimismo, Sánchez-Peña et al., (2006), citados por los mismos autores, determinaron una baja incidencia de mosca blanca (Bemisia tabaci).

Las plantas se encuentran a orilla de cercos, por lo que algunas poblaciones son erradicadas debido el avance de la frontera agrícola, incursión de ganado menor y aves, y ampliación de viviendas. Las poblaciones silvestres de tomate deben de ser estudiadas para clasificar materiales con buenas propiedades organolépticas y características genéticas que puedan ser aprovechadas, tanto por las familias como por los mejoradores, respectivamente. Nuez (1995); citado por Prohens et al., (2003), informa que especies endémicas de tomates (L. cheesmanii) en Galápagos, tienen características de interés para la mejora genética del tomate cultivado, tales como resistencia a salinidad, estrés hídrico, contenido de azúcares, caroteno, tolerancia a patógenos, articulación del pedicelo, entre otros.

El tomate (Lycopersicum spp.), se distribuye a lo largo de la RRGA y se encuentra asociado a especies silvestres de cucúrbitas, pasifloráceas, fabáceas, y poáceas (Benavides et al., 2010).

Las muestras de tomate silvestre de la RRGA varían en color (Figura 2), las tonalidades encontradas son combinaciones de amarillo, anaranjado y rojo, con mayor frecuencia rojo-naranja (9B8), rojo-amarillo (7B8), y naranjaamarillo (6A7). Estas variaciones obedecen al contenido de pigmentos licopeno y caroteno. Dichas tonalidades, también son reportadas por Prohens et al., (2003), en las especies $L$. typicum, L. minor, L. ceras., y L. pimp. De igual manera, Crisanto-Juárez et al. (2010), mencionan estos colores en tomates silvestres recolectados en Oaxaca, México.

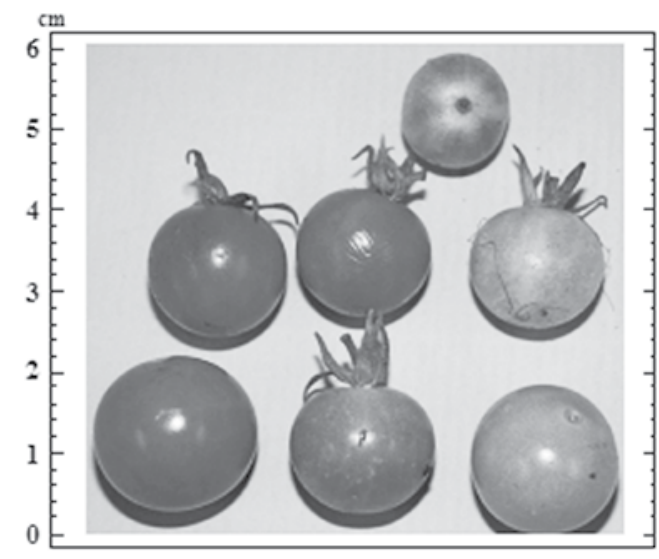

Figura 2. Variantes en tonalidades y longitud en $\mathrm{cm}$ de tomate silvestre de la RRGA.
Los materiales recolectados presentaron mayor variación cuando se expusieron a tratamientos, y fuera de su ambiente natural. A nivel general, los intervalos obtenidos fueron los siguientes: In Situ $=10.40 \pm 0.22$, Riego $=13.95 \pm 1.40$, y Biofertilizantes $=9.00 \pm 2.20$. Carrillo y Chávez (2010), reportan promedios en diámetro de 1.4 y $1.8 \mathrm{~cm}$, y pesos 1.5 a 3.0 g. Alvarez-Hernández et al., (2009), muestrearon frutos de tomates silvestres que presentaban promedios en diámetro de $1.05 \mathrm{~cm}$ a $1.22 \mathrm{~cm}$, y de $1.10 \mathrm{~cm}$ a $1.22 \mathrm{~cm}$ de longitud.

Tabla 2. Longitud del fruto en poblaciones de tomate silvestre

\begin{tabular}{|c|c|c|c|c|c|c|c|c|}
\hline & & P1 & P2 & P3 & P4 & P5 & P6 & Media \\
\hline \multirow[t]{2}{*}{ In situ } & & 13.20 & 15.62 & 13.89 & 13.84 & 14.15 & 13.87 & 14.10 \\
\hline & 0.4 & 13.89 & 12.22 & 12.73 & 12.88 & 14.01 & 7.84 & 12.26 \\
\hline \multirow[t]{3}{*}{ Riego } & 0.7 & 13.60 & 11.54 & 11.46 & 10.93 & 11.38 & 11.62 & 11.76 \\
\hline & 1.1 & 13.09 & 7.82 & 12.54 & 12.59 & 14.36 & 4.68 & 10.84 \\
\hline & 0 & 4.82 & 4.91 & 6.66 & 8.96 & 8.63 & 8.08 & 7.01 \\
\hline \multirow[t]{3}{*}{ Biofertilizantes } & 100 & 6.60 & 9.88 & 7.99 & 9.87 & 8.99 & 9.77 & 8.85 \\
\hline & 200 & 7.94 & 10.49 & 8.33 & 10.65 & 8.84 & 10.00 & 9.37 \\
\hline & 300 & 8.82 & 10.07 & 9.12 & 10.88 & 9.58 & 10.30 & 9.79 \\
\hline
\end{tabular}

Análisis físico-químico en tomate silvestre de Apacunca.

El fruto del tomate silvestre es una baya, con un sabor ligeramente ácido. El tomate presenta poca cantidad de calorías. La mayor parte de su peso es agua y el segundo constituyente en importancia son los hidratos de carbono (Tabla 3). Contiene azúcares simples que le confieren un ligero sabor dulce y algunos ácidos orgánicos que le otorgan el sabor ácido característico. El tomate es una fuente importante de ciertos minerales, como el potasio y el magnesio. De su contenido en vitaminas destacan la B1, B2, B5 y la vitamina C. Presenta también carotenoides como el licopeno (pigmento que da el color rojo característico al tomate). $\mathrm{La}$ vitamina $\mathrm{C}$ y el licopeno son antioxidantes con una función protectora del organismo (http://www.consumer.es).

Las fibras son los carbohidratos no digeribles y lignina que se encuentran intactos en las plantas; y las cenizas como los residuos inorgánicos de los alimentos que permanecen en la muestra posterior a la ignición u oxidación completa de la materia orgánica.

El tomate está entre los alimentos con bajo contenido en fibra $(<2 \mathrm{~g} / 100 \mathrm{~g}$ de alimento). En la Tabla 3, se muestran los valores por cada $100 \mathrm{~g}$, tanto en las variedades comerciales como en el tomate silvestre recolectado en la RRGA. Se puede observar que los análisis en cuanto a proteínas y agua son similares; no así en el contenido de grasa, que es casi nulo en el tomate silvestre nicaragüense. 
Tabla 3. Valor nutricional en crudo por cada $100 \mathrm{~g}$ de tomate Contenido Tomate comercial ${ }^{*}$ Tomate silvestre $^{* *}$

\begin{tabular}{lll}
\hline Agua & 95 & 92.43 \\
Calorías (Kcalorías) & 23 & -- \\
Proteínas & 0.8 & 0.78 \\
Grasas & 0.2 & 0.00 \\
Hidratos de Carbono & 3.5 & -- \\
Fibra & 1.8 & 0.72 \\
Cenizas & 0.6 & 1.47
\end{tabular}

Fuente: *USDA, http://www.consumer.es/, ** LABAL-MIFIC

Caracterización in situ de poblaciones de tomate silvestre. Mediante submuestreos in situ se encontró diferencias en las poblaciones. La variación en los caracteres fue baja. El peso promedio general fue de $1.84 \mathrm{~g}$, con mayor peso la población $\mathrm{P} 2$. En el tomate silvestre la relación entre ancho y longitud de fruto es aproximadamente de uno, lo que indica que los frutos son casi redondos. Las muestras de frutos no superaron los 2 $\mathrm{cm}$, y con mayor frecuencia se encontraron frutos de $1.5 \mathrm{~cm}$. El tomate silvestre de la RRGA, presentó entre 40 y 60 semillas por fruto, y aunque esta es una variable inapropiada para el consumo, no es relevante según los pobladores de la RRGA. Alvarez-Hernández et al., (2009), reportan intervalos de peso de fruto de $0.95 \pm 0.19,7.55 \pm 1.99,0.91 \pm 0.13,1.20 \pm 0.21$, $1.19 \pm 0.17$ y $8.87 \pm 2.01$ en seis poblaciones silvestres de tomate mexicanos. Los mismos autores, contabilizaron el número de semillas en frutos con los siguientes resultados: $44.75 \pm 5.76$, $\begin{array}{lll}104.1 \pm 30.17, & 42.05 \pm 36.20, & 42.05 \pm 7.56, \quad 36.20 \pm 7.88,\end{array}$ $37.35 \pm 7.91$ y $113.5 \pm 24.06$. Los valores promedios de la Tabla 4, contrastan con los muestreados por estos autores.

Tabla 4. Significación estadística en variables de fruto evaluadas en germoplasma de tomate silvestre en la RRGA, Somotillo, Chinandega

\begin{tabular}{|c|c|c|c|c|c|c|}
\hline Muestra & $\begin{array}{l}\text { Peso de } \\
\text { Fruto }\end{array}$ & $\begin{array}{l}\text { Diámetro } \\
\text { de fruto }\end{array}$ & $\begin{array}{l}\text { Longitud } \\
\text { de fruto }\end{array}$ & $\begin{array}{l}\text { Volumen } \\
\text { de fruto }\end{array}$ & Brixs & $\begin{array}{c}\text { Semilla por } \\
\text { fruto }\end{array}$ \\
\hline$\overline{\mathrm{P} 2}$ & $2.51 \mathrm{a}$ & $16.44 \mathrm{a}$ & $15.62 \mathrm{a}$ & $12.80 \mathrm{a}$ & $10.43 \mathrm{~d}$ & $50.00 \mathrm{~b}$ \\
\hline P5 & $1.90 \mathrm{~b}$ & $14.77 \mathrm{~b}$ & $14.15 \mathrm{~b}$ & $9.73 \mathrm{bc}$ & $10.60 \mathrm{~cd}$ & $48.80 \mathrm{~b}$ \\
\hline P3 & $1.75 \mathrm{c}$ & $14.65 \mathrm{~b}$ & $13.89 \mathrm{~b}$ & $10.37 \mathrm{~b}$ & $10.83 \mathrm{c}$ & 56.83 a \\
\hline P6 & $1.69 \mathrm{~cd}$ & $13.94 \mathrm{c}$ & $13.87 \mathrm{~b}$ & $10.33 \mathrm{~b}$ & $11.83 \mathrm{~b}$ & $48.13 \mathrm{~b}$ \\
\hline P4 & $1.63 \mathrm{~d}$ & $13.95 \mathrm{c}$ & $13.84 \mathrm{~b}$ & $8.73 \mathrm{~cd}$ & $12.47 \mathrm{c}$ & $43.73 \mathrm{c}$ \\
\hline P1 & $1.55 \mathrm{~d}$ & $13.64 \mathrm{c}$ & $13.13 \mathrm{c}$ & $8.73 \mathrm{~cd}$ & $10.83 \mathrm{c}$ & $47.33 \mathrm{bc}$ \\
\hline $\mathrm{R} 2$ & 0.96 & 0.91 & 0.88 & 0.85 & 0.98 & 0.80 \\
\hline Fisher & 0.002 & 0.012 & 0.001 & 0.01 & 0.045 & 0.023 \\
\hline $\mathrm{CV}$ & 4.42 & 2.47 & 2.81 & 9.25 & 1.17 & 4.95 \\
\hline
\end{tabular}

Promedios con letras similares no difieren (LSD, $\infty=0.05)$, CV Coeficiente de variación ( $\%)$.

Normas de riego $y$ biofertilizantes sobre poblaciones de tomate. El riego aplicado, así como las dosis de biofertilizante afectaron de manera significativa $(\mathrm{pr}=0.05)$, algunas variables. Las variables de frutos y tallo respondieron a las normas de riego y los niveles de biofertilizante, respectivamente (Tabla 5).

Tabla 5. Efecto normas de riego y biofertilizantes en poblaciones de tomate silvestre

\begin{tabular}{|c|c|c|c|c|c|c|c|c|c|c|c|c|}
\hline \multirow{2}{*}{$\begin{array}{l}\text { Variables } \\
\text { Ancho de hoja }\end{array}$} & \multirow{2}{*}{$\begin{array}{l}\text { Rep. } \\
0.476\end{array}$} & \multirow{2}{*}{$\begin{array}{c}\text { Pob Riego } \\
0.081\end{array}$} & \multicolumn{2}{|c|}{ Pob*Riego } & \multirow{2}{*}{$\begin{array}{l}\mathrm{R} 2 \\
0.68\end{array}$} & \multirow{2}{*}{$\begin{array}{l}\text { CV } \\
10.30\end{array}$} & \multirow{2}{*}{$\begin{array}{l}\text { Rep. } \\
0.818\end{array}$} & \multirow{2}{*}{$\begin{array}{l}\text { Pob } \\
0.000\end{array}$} & \multicolumn{2}{|c|}{ BiofPob*Biof } & \multirow{2}{*}{$\begin{array}{l}\mathrm{R} 2 \\
0.73\end{array}$} & \multirow{2}{*}{$\begin{array}{l}\mathrm{CV} \\
23.89\end{array}$} \\
\hline & & & 0.004 & 0.204 & & & & & 0.047 & 0.121 & & \\
\hline Longitud de la hoja & 0.211 & 0.008 & 0.188 & 0.083 & 0.71 & 10.50 & 0.375 & 0.000 & 0.030 & 0.009 & 0.86 & 16.67 \\
\hline Altura de tallo & 0.601 & 0.415 & 0.011 & 0.348 & 0.60 & 9.84 & 0.676 & 0.000 & 0.000 & 0.001 & 0.87 & 12.77 \\
\hline Diámetro de tallo & 0.326 & 0.018 & 0.447 & 0.812 & 0.68 & 10.73 & 0.593 & 0.001 & 0.001 & 0.160 & 0.75 & 17.52 \\
\hline Long.entrenudo & 0.578 & 0.266 & 0.948 & 0.526 & 0.62 & 11.09 & 0.068 & 0.000 & 0.002 & 0.001 & 0.81 & 16.05 \\
\hline Número de racimo & 0.173 & 0.214 & 0.017 & 0.031 & 0.72 & 31.10 & 0.884 & 0.050 & 0.034 & 0.843 & 0.59 & 48.20 \\
\hline Fruto maduro & 0.105 & 0.167 & 0.018 & 0.231 & 0.70 & 39.51 & 0.544 & 0.000 & 0.000 & 0.071 & 0.70 & 58.00 \\
\hline Fruto verde & 0.291 & 0.390 & 0.050 & 0.120 & 0.85 & 31.70 & 0.996 & 0.198 & 0.014 & 0.455 & 0.57 & 72.79 \\
\hline Frutos por planta & 0.162 & 0.665 & 0.058 & 0.261 & 0.65 & 37.13 & 0.864 & 0.005 & 0.132 & 0.832 & 0.45 & 72.84 \\
\hline Longitud de fruto & 0.758 & 0.090 & 0.438 & 0.379 & 0.80 & 6.70 & 0.947 & 0.003 & 0.179 & 0.027 & 0.73 & 20.13 \\
\hline Diámetro de fruto & 0.372 & 0.159 & 0.050 & 0.767 & 0.68 & 10.45 & 0.894 & 0.034 & 0.404 & 0.204 & 0.70 & 22.07 \\
\hline Peso de fruto & 0.577 & 0.066 & 0.013 & 0.627 & 0.65 & 19.43 & 0.376 & 0.489 & 0.390 & 0.433 & 0.60 & 85.00 \\
\hline Semilla por fruto & 0.632 & 0.021 & 0.068 & 0.968 & 0.60 & 15.04 & 0.531 & 0.007 & 0.393 & 0.583 & 0.65 & 26.70 \\
\hline Volumen de fruto & 0.853 & 0.019 & 0.020 & 0.825 & 0.66 & 23.83 & 0.688 & 0.005 & 0.178 & 0.719 & 0.62 & 61.75 \\
\hline Brix & 0.272 & 0.063 & 0.070 & 0.033 & 0.70 & 13.30 & 0.334 & 0.008 & 0.156 & 0.511 & 0.67 & 19.66 \\
\hline
\end{tabular}


La longitud de entrenudo promedio fue de $3.73 \mathrm{~cm}$ y $2.75 \mathrm{~cm}$ en el tomate silvestre de la RRGA para el efecto de riego y biofertilizante, respectivamente (Tabla 6); estos promedios coinciden con los reportados por Prohens et al., (2010) en L. typicum, (3.34 \pm 0.48$)$, y L. minor. (2.96 \pm 0.38$)$.

El peso promedio de fruto fue de 1.72 y $1.34 \mathrm{~g}$ para las normas de riego y niveles de biofertilizante. Prohens et al., (2010), reportan intervalos de $1.03 \pm 0.15$ para Gal red; y $0.43 \pm 0.12,0.35 \pm 0.03,0.77 \pm 0.19,5.41 \pm 1.5$ y $1.07 \pm 0.07$ en L. typicum, L. minor, L. ceras., y L. pimp, respectivamente.

Los grados Brixs reportados tuvieron un rango entre 4.29 y 5.72 (Tabla 6); Prohens et al., (2010), reportan intervalos de $10.37 \pm 0.88$ en L. typicum, $14.29 \pm 0.52$ en L. minor, $6.30 \pm 1.16$ en $L$. ceras, y $8.56 \pm 0.48$ en $L$. pimp

Tabla 6. Categorización estadística en normas de riego y niveles de biofertilizantes

\begin{tabular}{|c|c|c|c|c|c|c|c|c|c|c|}
\hline & & $\begin{array}{l}\text { Fruto } \\
\text { verde }\end{array}$ & $\begin{array}{l}\text { Fruto } \\
\text { maduro }\end{array}$ & $\begin{array}{l}\text { Peso de } \\
\text { fruto }\end{array}$ & $\begin{array}{l}\text { Longitud } \\
\text { fruto fruto }\end{array}$ & $\begin{array}{l}\text { Diámetro } \\
\text { fruto }\end{array}$ & $\begin{array}{l}\text { Semilla } \\
\text { de tallo }\end{array}$ & Altura & Brix & $\begin{array}{l}\text { Longitud de } \\
\text { entrenudos }\end{array}$ \\
\hline Riego & $\begin{array}{l}0.4 \\
0.7 \\
1.1\end{array}$ & $\begin{array}{l}12.6 \mathrm{a} \\
15.0 \mathrm{a} \\
12.4 \mathrm{a}\end{array}$ & $\begin{array}{l}12.2 \mathrm{~b} \\
17.3 \mathrm{a} \\
12.6 \mathrm{~b}\end{array}$ & $\begin{array}{l}1.9 \mathrm{a} \\
1.7 \mathrm{~b} \\
1.6 \mathrm{~b}\end{array}$ & $\begin{array}{l}12.6 \mathrm{a} \\
12.4 \mathrm{ab} \\
11.7 \mathrm{~b}\end{array}$ & $\begin{array}{c}13.8 \mathrm{a} \\
3.3 \mathrm{ab} \\
12.6 \mathrm{~b}\end{array}$ & $\begin{array}{l}44.5 \mathrm{a} \\
44.2 \mathrm{a} \\
39.8 \mathrm{~b}\end{array}$ & $\begin{array}{l}84.6 \mathrm{~b} \\
85.6 \mathrm{a} \\
77.4 \mathrm{~b}\end{array}$ & $\begin{array}{l}4.79 \mathrm{a} \\
4.79 \mathrm{a} \\
4.29 \mathrm{~b}\end{array}$ & $\begin{array}{l}3.7 \mathrm{a} \\
3.8 \mathrm{a} \\
3.7 \mathrm{a}\end{array}$ \\
\hline $\begin{array}{l}\text { Bioferti- } \\
\text { lizantes }\end{array}$ & $\begin{array}{c}0 \\
100 \\
200 \\
300\end{array}$ & $\begin{array}{r}2.6 \mathrm{~b} \\
12.1 \mathrm{a} \\
13.0 \mathrm{a} \\
10.7 \mathrm{a}\end{array}$ & $\begin{array}{l}14.3 \mathrm{~b} \\
16.9 \mathrm{ab} \\
25.4 \mathrm{a} \\
20.4 \mathrm{ab}\end{array}$ & $\begin{array}{l}2.7 \mathrm{a} \\
0.8 \mathrm{a} \\
0.9 \mathrm{a} \\
0.9 \mathrm{a}\end{array}$ & $\begin{array}{l}8.1 \mathrm{a} \\
8.5 \mathrm{a} \\
9.2 \mathrm{a} \\
9.1 \mathrm{a}\end{array}$ & $\begin{array}{l}8.5 \mathrm{a} \\
8.7 \mathrm{a} \\
9.5 \mathrm{a} \\
9.3 \mathrm{a}\end{array}$ & $\begin{array}{l}26.8 \mathrm{~b} \\
29.5 \mathrm{ab} \\
29.4 \mathrm{ab} \\
31.4 \mathrm{a}\end{array}$ & $\begin{array}{l}54.3 \mathrm{~b} \\
70.0 \mathrm{a} \\
77.6 \mathrm{a} \\
76.5 \mathrm{a}\end{array}$ & $\begin{array}{l}5.52 \mathrm{a} \\
5.72 \mathrm{a} \\
5.69 \mathrm{a} \\
4.97 \mathrm{a}\end{array}$ & $\begin{array}{l}2.4 \mathrm{~b} \\
2.8 \mathrm{a} \\
2.8 \mathrm{a} \\
3.0 \mathrm{a}\end{array}$ \\
\hline
\end{tabular}

Relación y variación de tratamientos. El análisis de componentes principales reflejó que la proporción acumulada en los dos componentes es suficiente para su interpretación (Figura 2). El primer componente (CP-1) aisló el 37.4\% de la variación total, y estuvo integrado por la longitud de fruto (0.381), ancho de fruto (0.375), longitud de entrenudos en el tallo (0.369), número de semillas por fruto (0.367) y altura del tallo (0.340). La variación aislada por el segundo componente (CP-2) fue de $26.3 \%$, y las variables discriminantes fueron, el número de racimos por planta (0.463), número de frutos por planta (0.450), número de frutos maduros (0.438) y el diámetro del tallo (0.369). Las variables de mayor peso en el CP-3 fueron el peso del fruto (-0.516) y los grados Brixs (-451). Dichas variables, acumularon el $67 \%, 74 \%$ y $47 \%$ a lo interno de los CP-1, CP-2 y CP-3.

Aunque la selección del número de componentes puede depender del objetivo del estudio e investigador; Hidalgo (2003) considera que un $70 \%$ de la variación fenotípica total aportada por los tres primeros componentes, es considerable.

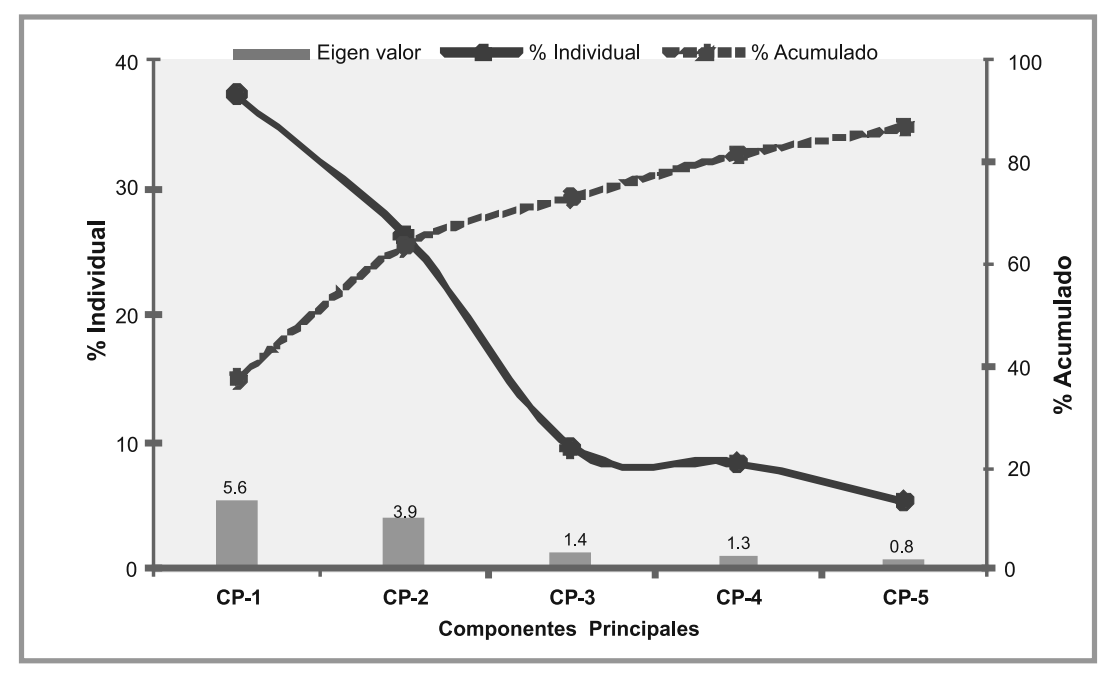

Figura 2. Proporción de varianzas en tratamientos de tomate silvestre recolectado en la RRGA. 
El análisis de componentes principales, aisló la variación total fenotípica en $63.6 \%$ y $73.2 \%$, en los dos y tres primeros componentes, respectivamente. Las variables ancho del fruto, longitud de entrenudos en el tallo y número de semillas en el fruto, fueron las características con mayor valor descriptivo en cuanto a la diferenciación de las muestras in situ (Figura 3) del resto de los tratamientos. Los tratamientos graficados en la Figura 4, reflejan el mismo comportamiento.

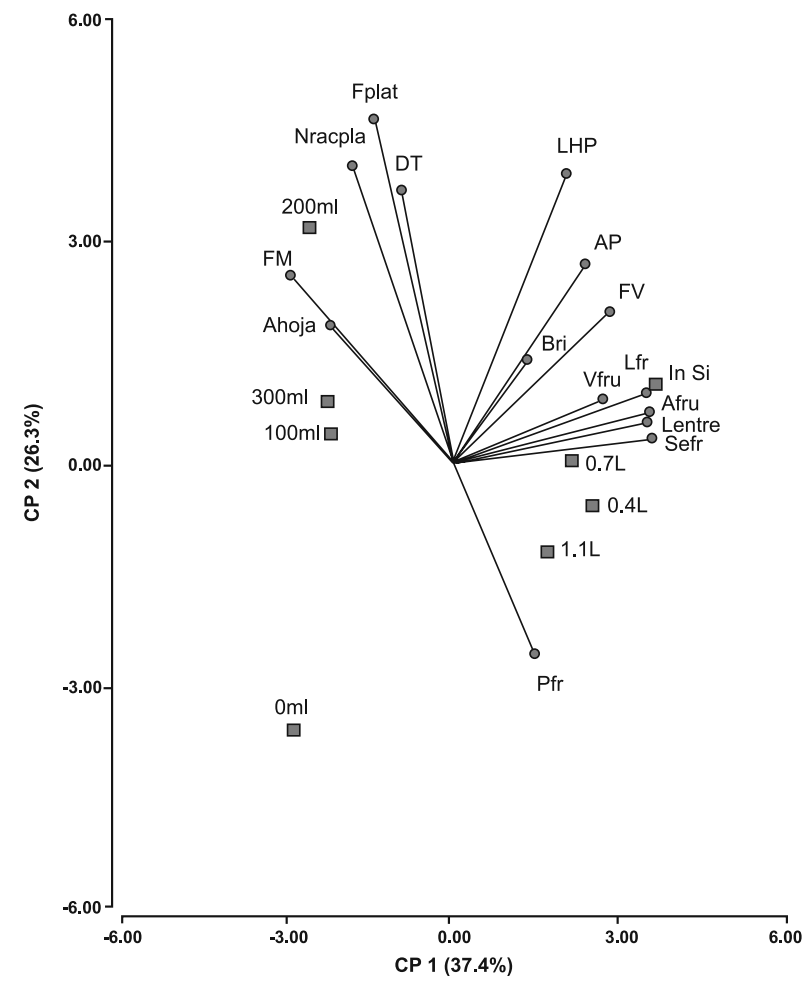

Figura 3. Distribución de tratamientos en tomate silvestre muestreados in situ y ex situ.

Las poblaciones evaluadas in situ, con normas de riego y niveles de biofertilizante conformaron tres núcleos bien diferenciados. Las mediciones realizadas en las poblaciones en su hábitat natural fueron menos variantes, en comparación a las conformadas ex situ y expuestas a tratamientos.

El análisis de conglomerados mostró que las poblaciones evaluadas in situ, con normas de riego y niveles de biofertilizante presentaron tres grupos muy diferentes a una distancia de 0.89 mediante el método Ward y la distancia de Gower con un coeficiente cofenético de 0.90. Las mediciones realizadas a las poblaciones in situ presentaron menor variación, en relación a las conformadas ex situ y expuestas a tratamientos (Figura 4).

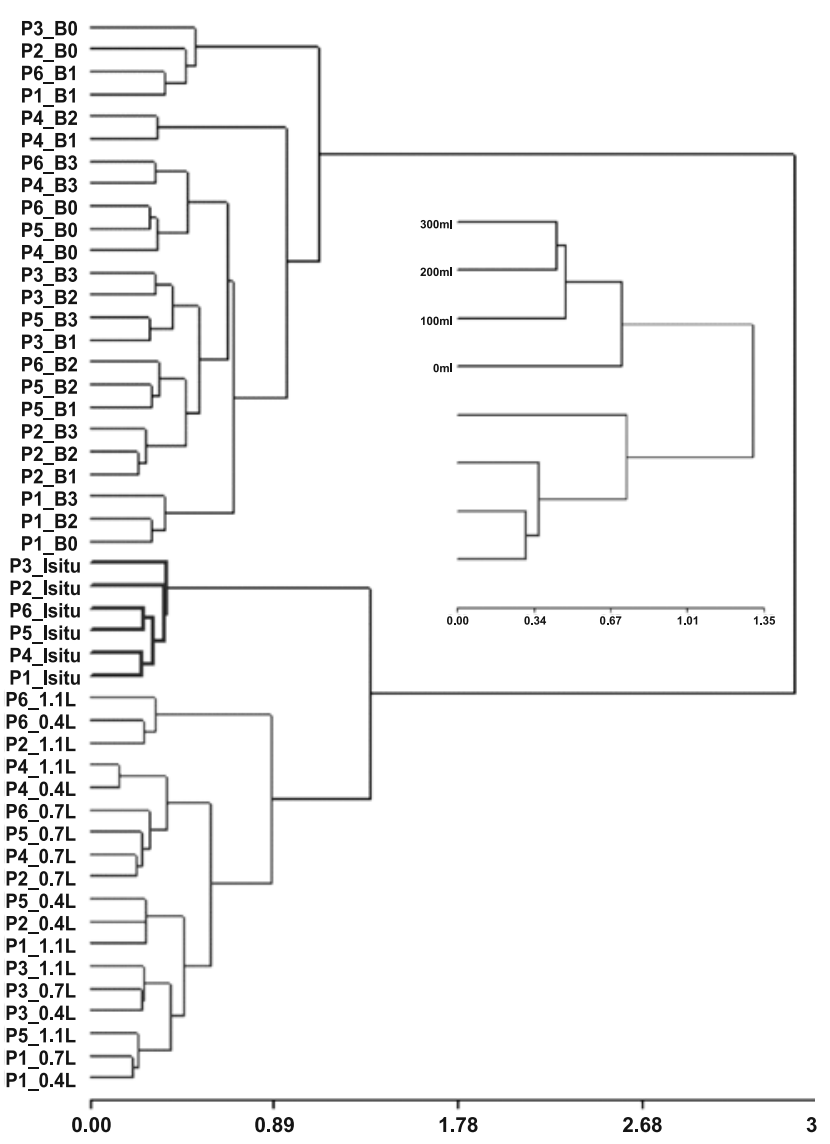

Figura 4. Relación en poblaciones de tomates silvestres muestreados in situ y ex situ.

\section{CONCLUSIONES}

Basado en el presente estudio sobre tomate silvestre, se realizaron las siguientes consideraciones:

La aplicación de niveles de riego y biofertilizante sobre las poblaciones ejercen efecto significativo en variables. La mayor cantidad de frutos se obtuvieron con la dosis intermedia de riego, y mayores niveles de biofertilizante.

Más del $70 \%$ de la variación total fue explicada por el fruto, longitud de tallo y entrenudos en la planta. Las poblaciones in situ, se distanciaron de las poblaciones con normas de riego y biofertilizantes. En su hábitat natural las poblaciones de tomate fueron menos variantes, en comparación a las conformadas ex situ.

El tomate silvestre de la RRGA tiene características organolépticas que pueden ser utilizadas de manera directa en la alimentación de las familias rurales, por consiguiente, se debe asegurar su conservación. 


\section{REFERENCIAS BIBLIOGRÁFICAS}

Alvarez-Hernández JC; Cortez-Madrigal, H; García-Ruíz, I. 2009. Exploración y caracterización de poblaciones silvestres de jitomate (Solanaceae) en tres regiones de Michoacaán, México. Polibotánica, Núm. 28, agostoseptiembre 2009, pp 139-159.

Azurdia, C. 1996a. Conservación in situ de los parientes silvestres de las plantas cultivadas. Lecturas en Recursos Fitogenéticos. Subprograma de Recursos Genéticos Vegetales (REGEVE). Instituto de Investigaciones Agronómicas. Facultad de Agronomía. USAC. p. 47-48.

Azurdia, C. 1996b. Las malezas como un reservorio genético de las plantas. Lecturas en Recursos Fitogenéticos. Subprograma de Recursos Genéticos Vegetales (REGEVE). Instituto de Investigaciones Agronómicas. Facultad de Agronomía. USAC. p. 12-13.

Benavides GA; Cisne C, J; Querol L, D. 2010. Rescate, conservación y manejo sostenible del teocintle de Nicaragua (Zea nicaraguensis ILTIS \& BENZ). Informe DRP. UNA-FAO. Managua, Nicaragua, 109 p.

Benavides G, A. 2002. Caracterización y evaluación ex situ de una población de teocintle anual (Zea nicaraguensis ILTIS \& BENZ) recolectada en el norte de Chinandega. Revista LA CALERA. Universidad Nacional Agraria. Año 2. No. 2. Agosto-2002. p. 6-13.

Carrillo R, J; Chávez J, LS. 2010. Caracterización agromorfológica de muestras de tomate de Oaxaca. Rev. Fitotec. Méx. Vol. 33 (Núm. Especial 4): 1-6.

Crisanto-Juárez A; Vera-Guzmán, A; Chávez-Servia, J; Carrillo-Rodríguez, J. 2010. Calidad de frutos de tomates silvestres (Licopersicon esculentum var. Cerasiforme Dunal) de Oaxaca, México. Rev. Fitotec. Méx. Vol. 33 (Núm. Especial 4): 7-13.

Hidalgo R. 2003. Variabilidad genética caracterización de especies vegetales. En Análisis estadístico de datos de caracterización morfológica de Recursos Fitogenéticos, Franco T. e Hidalgo R. (eds.). Boletín Técnico no. 8, Instituto Internacional de Recursos Fitogenéticos (IPGRI), Calí, Colombia, p. 2-26.

INETER. 2010. Datos climatológicos de Nicaragua.

IPGRI. 2000. Frutales del trópico americano, de la información a la investigación. Boletín de las Américas. Calí, Colombia. V. 6, No. 1. p. 4-8.

Kornerup, Andreas. 1984. Metthuen Handbook of Color. 252 p.

Levard, L; Marín, Y; Pérez, F; Ruíz, A; Serra, HL. 2000. Potenciales y limitantes para el desarrollo agropecuario en el municipio de Somotillo. Cuaderno de Investigación \# 10. NITLAPLAN-UCA/FIDA. 148 p.

MAG. 1997. Nicaragua, Potencialidades y Limitaciones de sus Territorios. Gobierno de Nicaragua. 170 p.

MAG-FOR. 1999. Regionalización Biofísica para el desarrollo Agropecuario del departamento de Chinandega. Managua, Nicaragua. $162 \mathrm{p}$.

Martin, GJ. 2001. Etnobotánica. Pueblos y Plantas, Manual de Métodos, Edit. Nordan-Comunidad, Montevideo, Uruguay. $240 \mathrm{p}$

Meyrat A. 2001. Estrategia nacional de biodiversidad Nicaragua. Estado de conservación de los ecosistemas PNUDNIC, MARENA. 189. $22 \mathrm{p}$.

MARENA (Ministerio del Ambiente y Recursos Naturales). 1999. Biodiversidad en Nicaragua: Un estudio de país. MARENA-PANIF. 1a. Ed. 469 p.

Prohens, J; Blanca, JM; Nuez, F. 2003. Caracterización de tomates silvestres de las Islas Galápagos. 114 actas de Horticultura No.39. X Congreso Nacional de Ciencias Hortícolas, Pontevedra 2003, Biodiversidad, 3 p. http:// www.comav.upv.es/publicomav/744.pdf.

USDA APHIS. 1991. Environmental Assessment and Finding of No Significant Impact on Tomato Containing an Antisense Polygalacturonase Gene. Permit Number 91-268-01. 